\title{
Homologue Pairing in Flies and Mammals: Gene Regulation When Two Are Involved
}

\author{
Manasi S. Apte and Victoria H. Meller \\ Department of Biological Sciences, Wayne State University, Detroit, MI 48202, USA \\ Correspondence should be addressed to Victoria H. Meller, vmeller@biology.biosci.wayne.edu \\ Received 27 June 2011; Revised 17 September 2011; Accepted 26 September 2011 \\ Academic Editor: Douglas M. Ruden
}

Copyright ( $\odot 2012$ M. S. Apte and V. H. Meller. This is an open access article distributed under the Creative Commons Attribution License, which permits unrestricted use, distribution, and reproduction in any medium, provided the original work is properly cited.

\begin{abstract}
Chromosome pairing is usually discussed in the context of meiosis. Association of homologues in germ cells enables chromosome segregation and is necessary for fertility. A few organisms, such as flies, also pair their entire genomes in somatic cells. Most others, including mammals, display little homologue pairing outside of the germline. Experimental evidence from both flies and mammals suggests that communication between homologues contributes to normal genome regulation. This paper will contrast the role of pairing in transmitting information between homologues in flies and mammals. In mammals, somatic homologue pairing is tightly regulated, occurring at specific loci and in a developmentally regulated fashion. Inappropriate pairing, or loss of normal pairing, is associated with gene misregulation in some disease states. While homologue pairing in flies is capable of influencing gene expression, the significance of this for normal expression remains unknown. The sex chromosomes pose a particularly interesting situation, as females are able to pair X chromosomes, but males cannot. The contribution of homologue pairing to the biology of the $\mathrm{X}$ chromosome will also be discussed.
\end{abstract}

\section{Introduction}

One of the most intriguing aspects of somatic homologue pairing is that such a basic condition has enormous variability between species. Homologues pair vigorously in Drosophila, as illustrated by the remarkable alignment of polytene chromosomes. In fact, homologue pairing is pervasive throughout the Diptera, but in other organisms the occurrence and extent of homologue pairing is often unknown [ 1 , 2 ]. Close association of homologous chromosomes in vegetative diploid budding yeast has been reported, but a careful reexamination suggested that little, if any, pairing occurs [3]. In diploid fission yeast both homologues occupy the same chromosome territory and centromeric pairing is observed in most cells [4]. Early studies suggested somatic homologue pairing in numerous plant species (Reviewed in [2]). Recent work supports the idea of homologue pairing in some grains and fungi, but also casts doubt on other reports of pairing in plants [5-8].

\section{Mammals: Pairing to Share Information}

Mammals have perhaps the most elaborate manifestation of homologue pairing. While complete pairing of the mammalian genome is not reported outside of the germline, somatic pairing of specific chromosomal regions does occur, but is tightly regulated. For example, homologous association of pericentromeric regions of human chromosome 1 is detected in cerebellar, but not cerebral, tissue [9]. Heterochromatic regions of chromosomes 8 and 17 also pair in parts of the brain (Figure 1(a)) [10,11]. Chromosome-specific pairing of chromosome 7 and 10 is also seen in case of cell line derived from follicular lymphoma [12]. Several cell lines derived from renal carcinomas display an abnormal pairing of one arm of chromosome 19 and misexpress genes within the paired region (Figure 1(b)) [13]. This suggests that modulation of homologue associations may be necessary for normal gene regulation. The mechanism of pairing in these examples has not been investigated. However, this type of pairing is 


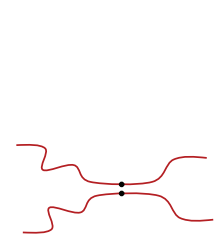

(a)

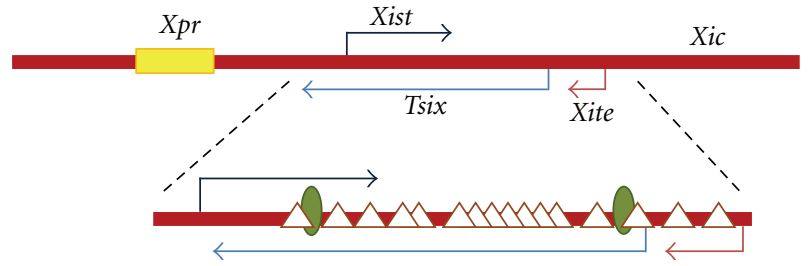

(d)

Figure 1: Modes of somatic pairing in mammalian tissues. (a) Pericentromeric homologue pairing in parts of the brain. Centromeres are depicted by black dots. (b) Abnormal pairing of chromosome $19 \mathrm{q}$ in renal carcinoma. (c) Looping between two sites on a chromosome (left) and interchromosomal contacts (right) are mediated by sequence-specific DNA-binding proteins such as CTCF (triangle) and cohesin (brown circle). (d) Pairing of the $X$ inactivation center $(X i c)$ initiates $\mathrm{X}$ chromosome inactivation in females. Sequences that participate in Xic pairing are depicted. The X-pairing region (Xpr, yellow) initiates Xic pairing. Tsix (light blue) and Xite (pink) pair transiently, enabling counting and choice to occur. Oct4 and CTCF are necessary for contact and communication at the Xic. Oct4-binding sites (green ovals) and CTCF-binding sites (triangles) within the Tsix and Xite regions of the mouse Xic are depicted.

very tissue specific and limited to portions of particular chromosomes. It therefore must depend on chromosome-specific features, as well as developmental cues.

The best understood somatic homologue associations in mammalian cells are transient and occur at individual loci, rather than encompassing extensive chromosomal regions. These contacts appear to be a subset of long-range interactions between chromosomes, which includes looping and interactions between nonhomologous regions (Figure 1(c)) $[14,15]$. One notable function of these interactions is their role in establishing inactivation of one of the two female $\mathrm{X}$ chromosomes and in controlling monoallelic expression of imprinted genes.

The long-range contacts made by mammalian homologues overlay a general nuclear organization that seems designed to discourage interaction. Mammalian chromosomes occupy nonoverlapping regions, termed chromosome territories, in the nucleus. These territories are organized by specific rules (Reviewed by Spector [16]). For example, genepoor regions tend to be close to the nuclear membrane, while gene-dense chromosomes localize in interior of the nucleus $[14,17]$. The territories of small and early replicating chromosomes also tend to be interior. Interestingly, in human epithelial cancer cell lines and mouse primary lymphocytes the territories occupied by the homologues are more widely separated than expected from a random distribution $[18,19]$.
One function of chromosome territories may be to keep the homologues apart.

The properties of the molecules that mediate long-range contacts between allelic and nonallelic loci suggest strategies that facilitate specific interactions. One of these molecules is CTCF (CCCTC-binding factor), a highly conserved, DNAbinding protein with a multitude of seemingly disparate regulatory functions (Reviewed by Philips and Corces [20]). Depending on context and binding partners, CTCF can be a transcriptional repressor or an activator [21-24]. Adjacent CTCF binding sites are often drawn into chromatin loops, insulating promoters from nearby regulatory regions [2530]. One of the best-understood examples is found at the imprinted Igf2/H19 locus. Imprinting, established in the parental germline, produces an allele-specific difference in genetic properties (Reviewed by Verona et al. [31]). The Igf2/ H19 locus has a CTCF-binding site that is differentially methylated in the parental germlines [32-34]. Methylation of the paternal allele blocks CTCF binding, preventing formation of an insulator that would otherwise separate Igf2 from an enhancer [33, 35-37]. On the maternal allele, CTCF binds between Igf2 and this enhancer, silencing Igf2 by insulation and through recruitment SUZ12, a member of the Polycomb Repressive Complex 2 (PRC2) [29]. On the maternal chromosome CTCF binding adjacent to $\mathrm{H} 19$ is necessary to induce expression of this transcript [38].

CTCF also mediates interactions between Igf2/H19, on chromosome 7, and other regions throughout the genome. Igf2/H19 contacts the Wsb1/Nf1 locus on chromosome 11 $[26,39]$. This interaction is dependent upon binding of CTCF to the maternal Igf2/H19 allele and is required for monoallelic expression from Wsb1/Nf1. Additional interactions between Igf2/H19 and several other imprinted loci have been identified, and these findings are consistent with the idea that Igf2/H19 coordinates the epigenetic status of imprinted regions throughout the genome [40].

Some imprinted homologues pair transiently, an activity that may be necessary for normal developmental regulation. In lymphocytes, transient association at $15 \mathrm{q} 11-\mathrm{q} 13$ occurs in late $S$ phase [41]. This region is imprinted, containing several monoallelically expressed genes. Loss of expression, or lack of normal imprinting at this locus, causes Prader-Willi and Angelman syndromes, both of which display developmental and neurological abnormalities (Reviewd by Lalande [42]). Interestingly, lymphocytes from Prader-Willi and Angelman syndrome patients do not pair [41]. Homologue communication at 15q11-q13 may be a factor in normal brain development, as this locus pairs persistently in normal brain, but not in brains from patients with some autism-spectrum disorders [43].

Homologue pairing also plays a central role in orchestration of $\mathrm{X}$ inactivation in mammalian females. Mammalian females randomly inactivate one $\mathrm{X}$ chromosome, thus maintaining an equivalent ratio of $\mathrm{X}$ to autosomal gene products in both sexes $[44,45]$. Each cell of the early embryo counts the number of $\mathrm{X}$ chromosomes and inactivates all but one (Reviewed by Royce-Tolland and Panning [46]). Counting, and choice of the inactive $X$, relies on a transient pairing of the $X$ inactivation center $(X i c)$, a locus on the $\mathrm{X}$ chromosome 
(Figure 1(d)). Pairing is believed to enable XX cells to coordinate inactivation of a single $\mathrm{X}$ chromosome. Deletion of regions engaged in pairing led to skewed or chaotic $\mathrm{X}$ inactivation [47]. The process of pairing is complex, involving multiple elements within the Xic. The X-pairing region $(X p r)$ may support initial interactions, and its deletion diminishes Xic pairing $[48,49]$. Several genes within the Xic produce noncoding RNAs that participate in counting and inactivation of the $\mathrm{X}$ chromosome. Xist, a long noncoding RNA, initiates the process of $\mathrm{X}$ inactivation and coats the inactive $\mathrm{X}$ (Reviewed by Chow and Heard [50]). Tsix, transcribed antisense to Xist, and a nearby gene Xite contribute to pairing of the Xic and also produce noncoding RNAs (Reviewed by Lee [51]). Following pairing, transcription of Tsix and Xite is necessary for orderly $\mathrm{X}$ inactivation, suggesting that communication might occur by an RNA-protein bridge between two $\mathrm{X}$ chromosomes [52]. CTCF plays a central role in pairing at the Xic. The Tsix promoter contains numerous CTCF binding sites (Figure 1(d)) [52-55]. Pairing at the Xic is disrupted upon the loss of CTCF [56]. Initiation of inactivation occurs during a narrow window in early development [57]. Oct4, a transcription factor key to the maintenance of stem cells, forms a complex with CTCF at Tsix, and is required for transient association of Xics [56]. After this transient pairing, the $\mathrm{X}$ chromosomes separate, assume different fates and localize to distinct nuclear compartments.

The examples above illustrate the idea that CTCF fulfills disparate functions in a developmental and cell type-specific manner. The proteins mentioned above, Oct4 and SUZ12, are among many CTCF partners that enable modulation of CTCF effects [58]. An additional CTCF binding protein that contributes to its localization and function is nucleophosmin, a component of the nucleolus [59]. Some loci that bind CTCF are anchored at the nucleolus, leading to the idea that the nucleolus functions as a hub where long-range interactions occur. While recruitment to the nucleolus appears to be a factor for some CTCF-bound loci, it does not contribute to $\mathrm{X}$ chromosome pairing $[59,60]$.

Another protein that contributes to CTCF function is cohesin, a multisubunit complex that regulates sister chromatid cohesion during meiosis and mitosis. Cohesin, consisting of SMC1, SMC3, Scc1, and Scc3 subunits, is believed to encircle sister chromatids to maintain their association $[61,62]$. The C-terminus of CTCF interacts with the cohesin subunit Scc3, and cohesin and CTCF are often colocalized on mammalian chromosomes [63-65]. Depletion of CTCF results in loss of cohesin binding but, at most sites, loss of cohesin does not affect CTCF binding to DNA [66, 67]. CTCF thus appears to recruit cohesin to specific DNA sequences. Cohesin recruitment facilitates long-range interactions, either by securing aligned regions or by inducing looping. For example, cohesin plays a regulatory role in CTCFmediated intrachromosomal contacts between sites in the interferon- $\gamma$ locus $[65,66]$. Loss of cohesin or CTCF also leads to misregulation of expression from Igf2/H19 [39, 64].

While cohesin colocalizes with CTCF on mammalian chromosomes, the association of these molecules is not universal. In Drosophila, cohesin and CTCF have not yet been shown to colocalize. In spite of this, in flies CTCF performs many functions similar to those in mammals. For example, it localizes to insulators and contributes to looping between boundary elements $[68,69]$. Drosophila CTCF also plays a role in imprinting in flies [70].

\section{Flies: Always in Touch}

In contrast to the carefully orchestrated pairing of specific loci in mammals, complete homologue pairing is the default condition in Drosophila. Pairing is evident from the mitotic cycle 13 of embryogenesis onwards [71, 72]. Cellularization occurs during cycle 14 , which marks a dramatic reorganization of the nucleus [73]. Heterochromatin becomes detectable at cycle 14, and transcription of zygotic genes begins in earnest [74]. While pairing is persistent throughout the cell cycle from this point onwards, it is relaxed, but still apparent, during replication and mitosis $[75,76]$.

Homologues might encounter each other by directed movement, or by random diffusion [77]. Analysis of chromosomal movements preceding pairing in embryos supports the idea that random motion leads to homologue encounters and suggests independent initiation at numerous sites, rather than a processive zippering along the length of the chromosome [71, 75]. Space constraints within a chromosome territory or an underlying chromosome arrangement could speed the search. Early studies by Rabl and Boveri revealed the nonrandom organization of the interphase nucleus. The centromeres cluster at one pole of the nucleus, while the chromosome arms extend across the nucleus towards the other pole. This polarized pattern of chromosomal arrangement, known as Rabl configuration, is not apparent in some species (rice, maize, mouse, and humans) but is observed in a wide range of organisms (S. cerevisiae, S. Pombe, Drosophila, and several grains) (Reviewed by Spector [16] and Santos and Shaw [78]). The Rabl configuration is reminiscent of the arrangement of chromosomes following mitosis, where the centromeres lead the chromosomes into the daughter cells. While the anaphase movement of chromosomes does promote this arrangement, cell division is not essential for the Rabl conformation in yeast [79]. Regardless of how formed, homologous chromosomes in the Rabl configuration are roughly aligned, more or less parallel, placing alleles closer together than predicted by chance distribution.

While pairing of imprinted loci and the Xic is necessary for correct regulation of developmentally important genes in mammals, there are no examples of flies utilizing chromosome pairing to count $\mathrm{X}$ chromosomes or to regulate monoallelic gene expression. However, homologue pairing in flies does affect gene expression through a mechanism known as transvection [80]. Pioneering work by Lewis on the Ultrabithorax $(U b x)$ gene showed that the mutant phenotype was stronger when pairing between two loss-of-function $U b x$ alleles was disrupted by chromosomal re-arrangements. When paired, $U b x$ expression was elevated, enabling complementation between the two mutations. A well-supported model for transvection is that pairing enables regulatory elements on one chromosome to drive (or silence) expression from an intact promoter on the other chromosome [81]. Confirmation of transvection is obtained when the phenotype is 
sensitive to disruption of pairing, for example, by inversion of one chromosome $[80,82]$. Transvection has been demonstrated for numerous genes in Drosophila, and it appears able to operate throughout the genome [83]. Transvection has also been observed in the diploid stages of Neurospora [5]. A few examples of transvection have been described in mammals, and the term is often used to describe nonallelic regulatory interactions in trans, such as the CTCF-mediated longrange interactions that were described in preceding sections $[84,85]$.

A limitation of our understanding of transvection is how alleles communicate. Communication may differ from gene to gene. For example, transvection at $U b x$ is disrupted by breaks anywhere within a large critical region between $U b x$ and the centromere, but transvection at the yellow gene is only sensitive to breaks very close to the gene. This is consistent with different mechanisms of pairing or communication at these loci, but could also reflect the length of the cell cycle, and thus the time available for homologue association, at the time of gene expression [86]. For example, expression of $U b x$ is required in rapidly cycling embryonic cells. In contrast, the critical period for yellow expression is in pupal cells that have ceased dividing. In accordance with this idea, extension of the cell cycle in $U b x$ mutants with inversions reduces phenotypic severity, presumably by allowing extended time for chromosome pairing [86].

One molecule that affects pairing-dependent gene regulation is encoded by zeste $(z)$. Zeste is a DNA-binding protein that affects pairing-dependent expression at many genes that display transvection (Reviewed by Pirrotta [87] and Duncan [88]). The Zeste protein polymerizes, leading to the suggestion that it might bridge homologues, but loss of Zeste does not affect homologue pairing [89]. Zeste binding sites are found in promoters, and the Zeste protein interacts with the activating Trithorax chromatin regulatory complex, as well as the repressing Polycomb PRC1 complex $[90,91]$. Thus it appears likely that Zeste is a transcription factor able to interpret the state of homologue pairing.

An RNAi screen in tissue culture cells identified Topoisomerase II (Top2) as necessary player in homologue pairing [76]. Topoisomerases play pivotal roles by solving topological problems associated with DNA replication, transcription, recombination, repair, and chromosome segregation (Reviewed by Nitiss [92]). Type II topoisomerases introduce double-strand breaks, pass an intact DNA duplex through the cut, and rejoin the cut ends. Top2 also makes up a large fraction of the insoluble nuclear matrix and contributes to chromosome architecture $[93,94]$. It preferentially binds scaffold-associated regions, which anchor chromatin loops during interphase. There are several potential mechanisms through which Top2 might contribute to pairing. Because it plays a central role in chromosome organization, loss of Top2 could lead to a general disruption that abrogates homologue association. It is also possible that Top2 engages in protein/ protein interactions that stabilize pairing.

One protein that interacts with Top2 and also affects pairing in Drosophila, is condensin. Condensins function in chromosome condensation, induction of DNA supercoiling, and anaphase chromosome segregation. Metazoans have two paralogous condensin complexes, condensin I and II. Each contains conserved SMC2 and SMC4 subunits, but different non-SMC subunits: Cap-H, Cap-G, and Cap-D2 or Cap-H2, Cap-G2, and Cap-D3 $[95,96]$. Condensins influence the activity of Top2, and Top2 interacts directly with the Drosophila Cap-H homologue Barren on mitotic chromosomes [97]. Both proteins are necessary for chromosome segregation, and loss of either produces a similar mitotic defect. Condensin I is also required for localization of Top2 on mitotic chromosomes in flies, yeast, and humans [98-100].

In spite of the dependent interactions between condensin and Top2, condensin acts to antagonize homologue pairing in Drosophila [101]. Most dramatically, ectopic expression of Cap-H2 in salivary glands separates the aligned polytene chromosomes. Increased condensin reduces transvection at two loci, revealing the dissociation of paired homologues in diploid cells. The involvement of Top2 and condensin reveals that homologue pairing in flies is regulated by conserved proteins necessary for the maintenance of chromosomal architecture and stability in all eukaryotic organisms. It will be fascinating to see if Top2 or condensin levels affect pairing in other organisms.

\section{Pairing and Sex Chromosomes}

An unanswered question is whether pairing-dependent regulation contributes to the expression of wild-type genes in Drosophila. Analysis of $U b x$ revealed that expression from a wild-type allele was increased when it could pair with a gain of function mutation [102]. Homologue pairing might also contribute to expression of other unmutated genes in a wildtype context. The phenotypic normality of flies with inverted chromosomes would suggest that transvection makes little contribution to expression, but a functional assay for homologue association demonstrated that alleles on inverted chromosomes can pair surprisingly efficiently, when given sufficient time [86]. But there are situations in which homologue pairing cannot occur, including the single male $\mathrm{X}$ chromosome and regions made hemizygous by deficiency. If pairing influences expression of wild type genes, the regulation of the entire $\mathrm{X}$ chromosome might differ between the sexes. This could contribute to sexually dimorphic expression or influence the biology of the X chromosome.

Flies have a dedicated regulatory system that accommodates hemizygosity of the X chromosome in males. Males produce the chromatin-modifying Male-Specific Lethal (MSL) complex, which is recruited to the $\mathrm{X}$ chromosome at $3 \mathrm{~h}$ after fertilization [103]. The result is increased expression of virtually every X-linked gene. Surprisingly, RNA sequencing of single-sexed embryos has identified partial dosage compensation at mitotic cycle 13, an hour before the MSL complex localizes to the X chromosome [104]. One mechanism proposed to explain this is that pairing of $\mathrm{X}$ chromatin in females inhibits transcription from X-linked genes. This idea deserves to be tested, as it could explain several situations in which dosage compensation occurs in the absence of the MSL complex. For example, $\mathrm{X}$-linked genes are dosage compensated in the male germline, where the MSL complex is not formed $[44,105]$. Autosomal deficiencies are partially 
compensated by an unknown mechanism [106]. In addition, considerable evidence supports the idea that the MSL complex does not fully compensate X-linked genes in somatic cells. If formation of the MSL complex is blocked, expression of X-linked genes is reduced by $25 \%-30 \%$, rather than the predicted 50\% [107, 108]. These observations support the idea that differences in gene copy number are buffered by mechanisms that operate throughout the genome (Reviewed by Stenberg and Larsson [106]).

A copy number buffering mechanism would differentially affect $\mathrm{X}$-linked gene expression in males and females. Over time, this could be a factor in creation of the striking differences in gene distribution observed when comparing the $\mathrm{X}$ chromosome and the autosomes in some species (Reviewed by Vicoso and Charlesworth [109] and Gurbich and Bachtrog [110]). For example, the mammalian X chromosome appears enriched for genes with a male-biased expression, including those expressed in the premeiotic testes [111]. This is postulated to reflect the fact that hemizygosity of the male X chromosome enables rapid selection for beneficial recessive alleles. The same argument should apply to other species with XY males, including flies. However, the X chromosomes of Drosophila melanogaster and related species are depleted for genes with male-biased expression in somatic tissues and testes and enriched for genes with female-biased expression [112]. These notable differences in the distributions of sex-biased genes in mammals and flies have yet to be adequately explained. A recent study revealed that the fly $\mathrm{X}$ chromosome was also depleted for developmentally regulated genes, with the notable exception of those expressed in the ovary [113]. The authors propose that demasculinization of the X chromosome was due in part to the fact that malebiased genes tend to be developmentally regulated and suggest that chromatin modification by the MSL complex may be incompatible with developmental regulation, making the $\mathrm{X}$ chromosome an unfavorable environment. However, a genome-wide buffering system that contributes to $\mathrm{X}$ chromosome dosage compensation could also influence the distribution of developmentally regulated genes. Analysis of expression in flies with autosomal deficiencies and duplications lends support to the idea that such a system exists, but constitutively expressed genes and those with highly regulated expression respond differently [114]. A speculative model for the role of homologue pairing in buffering gene dose is presented in Figure 2. A key feature of our model is that homologue pairing is repressive. The absence of pairing of the male X chromosome, and autosomal deficiencies, leads to a modest increase in expression from these regions.

\section{Conclusions}

Somatic chromosome pairing obeys strikingly different rules in mammals and flies. Mammals sharply limit contacts between homologues. When homologues do make contact it often serves to coordinate regulatory mechanisms, such as imprinting and $\mathrm{X}$ inactivation, that are essential for normal development. It seems ironic that mammals use pairing to communicate critical information, yet flies, with constant homologue pairing, appear to make little use of this feature

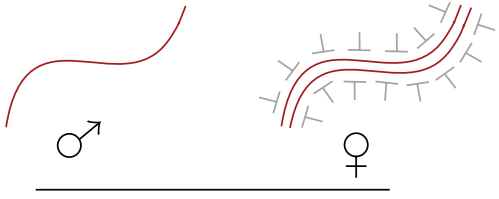

X chromosome

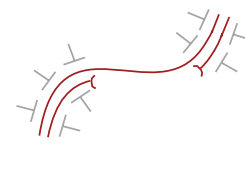

Deficiency

(c)
Figure 2: Hypothetical model for pairing-dependent buffering of gene dosage in flies. (a) The unpaired $\mathrm{X}$ chromosome of males escapes repression. (b) Paired female X chromosomes are subject to repression. (c) Paired regions of an autosome are repressed, but an unpaired region created by deficiency escapes repression.

of genome organization. Recent studies of early dosage compensation and buffering of copy number variation in flies suggest that additional regulatory mechanisms exist to accommodate variation in gene dosage. A pairing-based regulation of gene expression could account for many of the findings of these studies. A broader question is why homologue pairing exists in some species, but not in others. The precise control of homologue association in mammals, and inappropriate pairing in some cancers, suggests that homologue association can be dangerous. What this danger is, and how flies evade it, remains to be discovered.

\section{References}

[1] N. Stevens, "Study of the germ cells of certain Diptera, with reference to the Heterochromosomes and the Phenomena of Synapsis," Journal of Experimental Zoology, vol. 5, pp. 359374, 1908.

[2] C. W. Metz, "Chromosome studies on the Diptera. II. The paired association of chromosomes in the Diptera, and its significance," Journal of Experimental Zoology, vol. 21, pp. 213-279, 1916.

[3] A. Lorenz, J. Fuchs, R. Bürger, and J. Loidl, "Chromosome pairing does not contribute to nuclear architecture in vegetative yeast cells," Eukaryotic Cell, vol. 2, no. 5, pp. 856-866, 2003.

[4] H. Scherthan, J. Bähler, and J. Kohli, "Dynamics of chromosome organization and pairing during meiotic prophase in fission yeast," Journal of Cell Biology, vol. 127, no. 2, pp. 273285, 1994.

[5] R. Aramayo and R. L. Metzenberg, "Meiotic transvection in fungi," Cell, vol. 86, no. 1, pp. 103-113, 1996.

[6] J. B. Hollick, J. E. Dorweiler, and V. L. Chandler, "Paramutation and related allelic interactions," Trends in Genetics, vol. 13, no. 8, pp. 302-308, 1997.

[7] J. Bender, "Cytosine methylation of repeated sequences in eukaryotes: the role of DNA pairing," Trends in Biochemical Sciences, vol. 23, no. 7, pp. 252-256, 1998.

[8] A. J. Matzke, K. Watanabe, J. Van Der Winden, U. Naumann, and M. Matzke, "High frequency, cell type-specific visualization of fluorescent-tagged genomic sites in interphase and mitotic cells of living Arabidopsis plants," Plant Methods, vol. 6, article 2, 2010.

[9] E. P. Arnoldus, A. C. Peters, G. T. Bots, A. K. Raap, and M. Van Der Ploeg, "Somatic pairing of chromosome 1 centromeres in interphase nuclei of human cerebellum," Human Genetics, vol. 83, no. 3, pp. 231-234, 1989. 
[10] E. P. Arnoldus, A. Noordermeer, A. C. Peters, A. K. Raap, and M. Van Der Ploeg, "Interphase cytogenetics reveals somatic pairing of chromosome 17 centromeres in normal human brain tissue, but no trisomy 7 or sex-chromosome loss," Cytogenetics and Cell Genetics, vol. 56, no. 3-4, pp. 214-216, 1991.

[11] S. J. Dalrymple, J. F. Herath, T. J. Borell, C. A. Moertel, and R. B. Jenkins, "Correlation of cytogenetic and fluorescence in situ hybridization (FISH) studies in normal and gliotic brain," Journal of Neuropathology and Experimental Neurology, vol. 53, no. 5, pp. 448-456, 1994.

[12] N. B. Atkin and Z. Jackson, "Evidence for somatic pairing of chromosome 7 and 10 homologs in a follicular lymphoma," Cancer Genetics and Cytogenetics, vol. 89, no. 2, pp. 129-131, 1996.

[13] J. M. Koeman, R. C. Russell, M. H. Tan et al., "Somatic pairing of chromosome 19 in renal oncocytoma is associated with deregulated EGLN2-mediated [corrected] oxygen-sensing response," PLoS Genetics, vol. 4, no. 9, Article ID e1000176, 2008.

[14] T. Cremer and C. Cremer, "Chromosome territories, nuclear architecture and gene regulation in mammalian cells," Nature Reviews Genetics, vol. 2, no. 4, pp. 292-301, 2001.

[15] M. Bartkuhn and R. Renkawitz, "Long range chromatin interactions involved in gene regulation," Biochimica et Biophysica Acta, vol. 1783, no. 11, pp. 2161-2166, 2008.

[16] D. L. Spector, "The dynamics of chromosome organization and gene regulation," Annual Review of Biochemistry, vol. 72, pp. 573-608, 2003.

[17] J. A. Croft, J. M. Bridger, S. Boyle, P. Perry, P. Teague, and W. A. Bickmore, "Differences in the localization and morphology of chromosomes in the human nucleus," Journal of Cell Biology, vol. 145, no. 6, pp. 1119-1131, 1999.

[18] L. B. Caddle, J. L. Grant, J. Szatkiewicz et al., "Chromosome neighborhood composition determines translocation outcomes after exposure to high-dose radiation in primary cells," Chromosome Research, vol. 15, no. 8, pp. 1061-1073, 2007.

[19] C. Heride, M. Ricoul, K. Kiêu et al., "Distance between homologous chromosomes results from chromosome positioning constraints," Journal of Cell Science, vol. 123, no. 23, pp. 4063-4075, 2010.

[20] J. E. Phillips and V. G. Corces, "CTCF: master weaver of the genome," Cell, vol. 137, no. 7, pp. 1194-1211, 2009.

[21] V. V. Lobanenkov, R. H. Nicolas, V. V. Adler et al., "A novel sequence-specific DNA binding protein which interacts with three regularly spaced direct repeats of the CCCTC-motif in the $5^{\prime}$-flanking sequence of the chicken c-myc gene," Oncogene, vol. 5, no. 12, pp. 1743-1753, 1990.

[22] E. M. Klenova, R. H. Nicolas, H. F. Paterson et al., "CTCF, a conserved nuclear factor required for optimal transcriptional activity of the chicken c-myc gene, is an 11-Zn-finger protein differentially expressed in multiple forms," Molecular and Cellular Biology, vol. 13, no. 12, pp. 7612-7624, 1993.

[23] G. N. Filippova, S. Fagerlie, E. M. Klenova et al., "An exceptionally conserved transcriptional repressor, CTCF, employs different combinations of zinc fingers to bind diverged promoter sequences of avian and mammalian c-myc oncogenes," Molecular and Cellular Biology, vol. 16, no. 6, pp. 2802-2813, 1996.

[24] A. A. Vostrov and W. W. Quitschke, "The zinc finger protein CTCF binds to the APB $\beta$ domain of the amyloid $\beta$-protein precursor promoter: evidence for a role in transcriptional activation," Journal of Biological Chemistry, vol. 272, no. 52, pp. 33353-33359, 1997.
[25] A. Murrell, S. Heeson, and W. Reik, "Interaction between differentially methylated regions partitions the imprinted genes Igf2 and H19 into parent-specific chromatin loops," Nature Genetics, vol. 36, no. 8, pp. 889-893, 2004.

[26] S. Kurukuti, V. K. Tiwari, G. Tavoosidana et al., "CTCF binding at the $\mathrm{H} 19$ imprinting control region mediates maternally inherited higher-order chromatin conformation to restrict enhancer access to Igf2," Proceedings of the National Academy of Sciences of the United States of America, vol. 103, no. 28, pp. 10684-10689, 2006.

[27] E. Splinter, H. Heath, J. Kooren et al., "CTCF mediates longrange chromatin looping and local histone modification in the $\beta$-globin locus," Genes and Development, vol. 20, no. 17, pp. 2349-2354, 2006.

[28] C. Hou, H. Zhao, K. Tanimoto, and A. Dean, "CTCFdependent enhancer-blocking by alternative chromatin loop formation," Proceedings of the National Academy of Sciences of the United States of America, vol. 105, no. 51, pp. 2039820403, 2008.

[29] T. Li, J. F. Hu, X. Qiu et al., "CTCF regulates allelic expression of Igf2 by orchestrating a promoter-polycomb repressive complex 2 intrachromosomal loop," Molecular and Cellular Biology, vol. 28, no. 20, pp. 6473-6482, 2008.

[30] P. Majumder, J. A. Gomez, B. P. Chadwick, and J. M. Boss, "The insulator factor CTCF controls MHC class II gene expression and is required for the formation of long-distance chromatin interactions," Journal of Experimental Medicine, vol. 205, no. 4, pp. 785-798, 2008.

[31] R. I. Verona, M. R. Mann, and M. S. Bartolomei, "Genomic imprinting: intricacies of epigenetic regulation in clusters," Annual Review of Cell and Developmental Biology, vol. 19, pp. 237-259, 2003.

[32] K. D. Tremblay, J. R. Saam, R. S. Ingram, S. M. Tilghman, and M. S. Bartolomei, "A paternal-specific methylation imprint marks the alleles of the mouse H19 gene," Nature Genetics, vol. 9, no. 4, pp. 407-413, 1995.

[33] A. T. Hark, C. J. Schoenherr, D. J. Katz, R. S. Ingram, J. M. Levorse, and S. M. Tilghman, "CTCF mediates methylationsensitive enhancer-blocking activity at the H19/Igf2 locus," Nature, vol. 405, no. 6785, pp. 486-489, 2000.

[34] A. M. Fedoriw, P. Stein, P. Svoboda, R. M. Schultz, and M. S. Bartolomei, "Transgenic RNAi reveals essential function for CTCF in H19 gene imprinting," Science, vol. 303, no. 5655, pp. 238-240, 2004.

[35] A. C. Bell and G. Felsenfeld, "Methylation of a CTCF-dependent boundary controls imprinted expression of the Igf2 gene," Nature, vol. 405, no. 6785, pp. 482-485, 2000.

[36] C. Kanduri, V. Pant, D. Loukinov et al., "Functional association of CTCF with the insulator upstream of the H19 gene is parent of origin-specific and methylation-sensitive," Current Biology, vol. 10, no. 14, pp. 853-856, 2000.

[37] P. Szabó, S. H. Tang, A. Rentsendorj, G. P. Pfeifer, and J. R. Mann, "Maternal-specific footprints at putative CTCF sites in the H19 imprinting control region give evidence for insulator function," Current Biology, vol. 10, no. 10, pp. 607-610, 2000.

[38] C. J. Schoenherr, J. M. Levorse, and S. M. Tilghman, "CTCF maintains differential methylation at the Igf2/H19 locus," Nature Genetics, vol. 33, no. 1, pp. 66-69, 2003.

[39] J. Q. Ling, T. Li, J. F. Hu et al., "CTCF mediates interchromosomal colocalization between Igf2/H19 and Wsb1/Nf1," Science, vol. 312, no. 5771, pp. 269-272, 2006.

[40] K. S. Sandhu, C. Shi, M. Sjölinder et al., "Nonallelic transvection of multiple imprinted loci is organized by the H19 
imprinting control region during germline development," Genes and Development, vol. 23, no. 22, pp. 2598-2603, 2009.

[41] J. M. LaSalle and M. Lalande, "Homologous association of oppositely imprinted chromosomal domains," Science, vol. 272, no. 5262, pp. 725-728, 1996.

[42] M. Lalande, "Parental imprinting and human disease," Annual Review of Genetics, vol. 30, pp. 173-195, 1996.

[43] K. N. Thatcher, S. Peddada, D. H. Yasui, and J. M. LaSalle, "Homologous pairing of 15q11-13 imprinted domains in brain is developmentally regulated but deficient in Rett and autism samples," Human Molecular Genetics, vol. 14, no. 6, pp. 785-797, 2005.

[44] V. Gupta, M. Parisi, D. Sturgill et al., "Global analysis of Xchromosome dosage compensation," Journal of Biology, vol. 5, article 3, 2006.

[45] D. K. Nguyen and C. M. Disteche, "Dosage compensation of the active X chromosome in mammals," Nature Genetics, vol. 38, no. 1, pp. 47-53, 2006.

[46] M. Royce-Tolland and B. Panning, "X-inactivation: it takes two to count," Current Biology, vol. 18, no. 6, pp. R255-R256, 2008.

[47] J. T. Lee, "Homozygous Tsix mutant mice reveal a sex-ratio distortion and revert to random X-inactivation," Nature Genetics, vol. 32, no. 1, pp. 195-200, 2002.

[48] C. P. Bacher, M. Guggiari, B. Brors et al., "Transient colocalization of X-inactivation centres accompanies the initiation of X inactivation," Nature Cell Biology, vol. 8, no. 3, pp. 293299, 2006.

[49] S. Augui, G. J. Filion, S. Huart et al., "Sensing X chromosome pairs before $\mathrm{X}$ inactivation via a novel $\mathrm{X}$-pairing region of the Xic," Science, vol. 318, no. 5856, pp. 1632-1636, 2007.

[50] J. Chow and E. Heard, "X inactivation and the complexities of silencing a sex chromosome," Current Opinion in Cell Biology, vol. 21, no. 3, pp. 359-366, 2009.

[51] J. T. Lee, "Lessons from X-chromosome inactivation: long ncRNA as guides and tethers to the epigenome," Genes and Development, vol. 23, no. 16, pp. 1831-1842, 2009.

[52] N. Xu, M. E. Donohoe, S. S. Silva, and J. T. Lee, "Evidence that homologous X-chromosome pairing requires transcription and Ctcf protein," Nature Genetics, vol. 39, no. 11, pp. 13901396, 2007.

[53] W. Chao, K. D. Huynh, R. J. Spencer, L. S. Davidow, and J. T. Lee, "CTCF, a candidate trans-acting factor for X-inactivation choice," Science, vol. 295, no. 5553, pp. 345-347, 2002.

[54] N. Xu, C. L. Tsai, and J. T. Lee, "Transient homologous chromosome pairing marks the onset of X inactivation," Science, vol. 311, no. 5764, pp. 1149-1152, 2006.

[55] M. Xu and P. R. Cook, "The role of specialized transcription factories in chromosome pairing," Biochimica et Biophysica Acta, vol. 1783, no. 11, pp. 2155-2160, 2008.

[56] M. E. Donohoe, S. S. Silva, S. F. Pinter, N. Xu, and J. T. Lee, "The pluripotency factor Oct4 interacts with Ctcf and also controls X-chromosome pairing and counting," Nature, vol. 460, no. 7251, pp. 128-132, 2009.

[57] A. Wutz and R. Jaenisch, "A shift from reversible to irreversible $\mathrm{X}$ inactivation is triggered during ES cell differentiation," Molecular Cell, vol. 5, no. 4, pp. 695-705, 2000.

[58] J. A. Wallace and G. Felsenfeld, "We gather together: insulators and genome organization," Current Opinion in Genetics and Development, vol. 17, no. 5, pp. 400-407, 2007.

[59] T. M. Yusufzai, H. Tagami, Y. Nakatani, and G. Felsenfeld, "CTCF tethers an insulator to subnuclear sites, suggesting shared insulator mechanisms across species," Molecular Cell, vol. 13, no. 2, pp. 291-298, 2004.
[60] O. Masui, I. Bonnet, P. Le Baccon et al., "Live-cell chromosome dynamics and outcome of X chromosome pairing events during ES cell differentiation," Cell, vol. 145, no. 3, pp. 447-458, 2011.

[61] D. Ivanov and K. Nasmyth, "A physical assay for sister chromatid cohesion in vitro," Molecular Cell, vol. 27, no. 2, pp. 300-310, 2007.

[62] K. Nasmyth and C. H. Haering, "Cohesin: its roles and mechanisms," Annual Review of Genetics, vol. 43, pp. 525-558, 2009.

[63] V. Parelho, S. Hadjur, M. Spivakov et al., "Cohesins functionally associate with CTCF on mammalian chromosome arms," Cell, vol. 132, no. 3, pp. 422-433, 2008.

[64] K. S. Wendt, K. Yoshida, T. Itoh et al., "Cohesin mediates transcriptional insulation by CCCTC-binding factor," Nature, vol. 451, no. 7180, pp. 796-801, 2008.

[65] T. Xiao, J. Wallace, and G. Felsenfeld, "Specific sites in the C terminus of CTCF interact with the SA2 subunit of the cohesin complex and are required for cohesin-dependent insulation activity," Molecular and Cellular Biology, vol. 31, no. 11, pp. 2174-2183, 2011.

[66] S. Hadjur, L. M. Williams, N. K. Ryan et al., "Cohesins form chromosomal cis-interactions at the developmentally regulated IFNG locus," Nature, vol. 460, no. 7253, pp. 410-413, 2009.

[67] R. Nativio, K. S. Wendt, Y. Ito et al., "Cohesin is required for higher-order chromatin conformation at the imprinted IGF2-H19 locus," PLoS Genetics, vol. 5, no. 11, Article ID e1000739, 2009.

[68] E. E. Holohan, C. Kwong, B. Adryan et al., "CTCF genomic binding sites in Drosophila and the organisation of the bithorax complex," PLoS genetics, vol. 3, no. 7, p. e112, 2007.

[69] O. Kyrchanova, T. Ivlieva, S. Toshchakov, A. Parshikov, O. Maksimenko, and P. Georgiev, "Selective interactions of boundaries with upstream region of Abd-B promoter in Drosophila bithorax complex and role of dCTCF in this process," Nucleic Acids Research, vol. 39, pp. 3042-3052, 2011.

[70] W. A. MacDonald, D. Menon, N. J. Bartlett et al., "The Drosophila homolog of the mammalian imprint regulator, CTCF, maintains the maternal genomic imprint in Drosophila melanogaster," BMC Biology, vol. 8, article 105, 2010.

[71] J. C. Fung, W. F. Marshall, A. Dernburg, D. A. Agard, and J. W. Sedat, "Homologous chromosome pairing in Drosophila melanogaster proceeds through multiple independent initiations," Journal of Cell Biology, vol. 141, no. 1, pp. 5-20, 1998.

[72] Y. Hiraoka, A. F. Dernburg, S. J. Parmelee, M. C. Rykowski, D. A. Agard, and J. W. Sedat, "The onset of homologous chromosome pairing during Drosophila melanogaster embryogenesis," Journal of Cell Biology, vol. 120, no. 3, pp. 591-600, 1993.

[73] V. E. Foe and B. M. Alberts, "Studies of nuclear and cytoplasmic behavior during the five mitotic cycles that precede gastrulation in Drosophila embryogenesis," Journal of Cell Science, vol. 61, pp. 31-70, 1983.

[74] B. Y. Lu, J. Ma, and J. C. Eissenberg, "Developmental regulation of heterochromatin-mediated gene silencing in Drosophila," Development, vol. 125, no. 12, pp. 2223-2234, 1998.

[75] A. K. Csink and S. Henikoff, "Large-scale chromosomal movements during interphase progression in Drosophila," Journal of Cell Biology, vol. 143, no. 1, pp. 13-22, 1998.

[76] B. R. Williams, J. R. Bateman, N. D. Novikov, and C. T. Wu, "Disruption of topoisomerase II perturbs pairing in Drosophila cell culture," Genetics, vol. 177, no. 1, pp. 31-46, 2007. 
[77] P. R. Cook, "The transcriptional basis of chromosome pairing," Journal of Cell Science, vol. 110, no. 9, pp. 1033-1040, 1997.

[78] A. P. Santos and P. Shaw, "Interphase chromosomes and the Rabl configuration: does genome size matter?" Journal of Microscopy, vol. 214, no. 2, pp. 201-206, 2004.

[79] Q. W. Jin, J. Fuchs, and J. Loidl, "Centromere clustering is a major determinant of yeast interphase nuclear organization," Journal of Cell Science, vol. 113, no. 11, pp. 1903-1912, 2000.

[80] E. B. Lewis, "The theory and application of a new method of detecting chromosomal rearrangements in Drosphila melanogaster," American Naturalist, vol. 88, pp. 225-239, 1954.

[81] P. K. Geyer, M. M. Green, and V. G. Corces, "Tissue specific transcriptional enhancers may act in trans on the gene located in the homologous chromosome: the molecular basis of transvection in Drosophila," EMBO Journal, vol. 9, no. 7, pp. 2247-2256, 1990.

[82] S. A. Ou, E. Chang, S. Lee, K. So, C. T. Wu, and J. R. Morris, "Effects of chromosomal rearrangements on transvection at the yellow gene of Drosophila melanogaster," Genetics, vol. 183, no. 2, pp. 483-496, 2009.

[83] J. L. Chen, K. L. Huisinga, M. M. Viering, S. A. Ou, C. T. Wu, and P. K. Geyer, "Enhancer action in trans is permitted throughout the Drosophila genome," Proceedings of the $\mathrm{Na}$ tional Academy of Sciences of the United States of America, vol. 99, no. 6, pp. 3723-3728, 2002.

[84] M. Rassoulzadegan, M. Magliano, and F. Cuzin, "Transvection effects involving DNA methylation during meiosis in the mouse," EMBO Journal, vol. 21, no. 3, pp. 440-450, 2002.

[85] H. Liu, J. Huang, J. Wang et al., "Transvection mediated by the translocated cyclin D1 locus in mantle cell lymphoma," Journal of Experimental Medicine, vol. 205, no. 8, pp. 1843 1858, 2008.

[86] M. M. Golic and K. G. Golic, "A quantitative measure of the mitotic pairing of alleles in Drosophila melanogaster and the influence of structural heterozygosity," Genetics, vol. 143, no. 1, pp. 385-400, 1996.

[87] V. Pirrotta, "The genetics and molecular biology of zeste in Drosophila melanogaster," Advances in Genetics, vol. 29, pp. 301-348, 1991.

[88] I. W. Duncan, "Transvection effects in Drosophila," Annual Review of Genetics, vol. 36, pp. 521-556, 2002.

[89] M. J. Gemkow, P. J. Verveer, and D. J. Arndt-Jovin, "Homologous association of the Bithorax-Complex during embryogenesis: consequences for transvection in Drosophila melanogaster," Development, vol. 125, no. 22, pp. 4541-4552, 1998.

[90] A. J. Kal, T. Mahmoudi, N. B. Zak, and C. P. Verrijzer, "The Drosophila Brahma complex is an essential coactivator for the trithorax group protein Zeste," Genes and Development, vol. 14, no. 9, pp. 1058-1071, 2000.

[91] A. J. Saurin, Z. Shao, H. Erdjument-Bromage, P. Tempst, and R. E. Kingston, "A Drosophila Polycomb group complex includes Zeste and dTAFII proteins," Nature, vol. 412, no. 6847, pp. 655-660, 2001.

[92] J. L. Nitiss, "DNA topoisomerase II and its growing repertoire of biological functions," Nature Reviews Cancer, vol. 9, no. 5, pp. 327-337, 2009.

[93] S. M. Gasser, T. Laroche, J. Falquet, E. Boy De La Tour, and U. K. Laemmli, "Metaphase chromosome structure. Involvement of topoisomerase II," Journal of Molecular Biology, vol. 188, no. 4, pp. 613-629, 1986.

[94] Y. Adachi, E. Kas, and U. K. Laemmli, "Preferential, cooperative binding of DNA topoisomerase II to scaffold-associated regions," EMBO Journal, vol. 8, no. 13, pp. 3997-4006, 1989.
[95] T. Ono, A. Losada, M. Hirano, M. P. Myers, A. F. Neuwald, and T. Hirano, "Differential contributions of condensin I and condensin II to mitotic chromosome architecture in vertebrate cells," Cell, vol. 115, no. 1, pp. 109-121, 2003.

[96] F. M. Yeong, H. Hombauer, K. S. Wendt et al., "Identification of a subunit of a novel kleisin- $\beta / \mathrm{SMC}$ complex as a potential substrate of protein phosphatase 2A," Current Biology, vol. 13, no. 23, pp. 2058-2064, 2003.

[97] M. A. Bhat, A. V. Philp, D. M. Glover, and H. J. Bellen, "Chromatid segregation at anaphase requires the barren product, a novel chromosome-associated protein that interacts with topoisomerase II,” Cell, vol. 87, no. 6, pp. 1103-1114, 1996.

[98] N. Bhalla, S. Biggins, and A. W. Murray, "Mutation of YCS4, a budding yeast condensin subunit, affects mitotic and nonmitotic chromosome behavior," Molecular Biology of the Cell, vol. 13, no. 2, pp. 632-645, 2002.

[99] P. A. Coelho, J. Queiroz-Machado, and C. E. Sunkel, "Condensin-dependent localisation of topoisomerase II to an axial chromosomal structure is required for sister chromatid resolution during mitosis," Journal of Cell Science, vol. 116, no. 23, pp. 4763-4776, 2003.

[100] K. Maeshima and U. K. Laemmli, "A Two-step scaffolding model for mitotic chromosome assembly," Developmental Cell, vol. 4, no. 4, pp. 467-480, 2003.

[101] T. A. Hartl, H. F. Smith, and G. Bosco, "Chromosome alignment and transvection are antagonized by condensin II," Science, vol. 322, no. 5906, pp. 1384-1387, 2008.

[102] A. S. Goldsborough and T. B. Kornberg, "Reduction of transcription by homologue asynapsis in Drosophila imaginal discs," Nature, vol. 381, no. 6585, pp. 807-810, 1996.

[103] J. C. Lucchesi, "Dosage compensation in Drosophila and the 'complex' world of transcriptional regulation," BioEssays, vol. 18, no. 7, pp. 541-547, 1996.

[104] S. E. Lott, J. E. Villalta, G. P. Schroth, S. Luo, L. A. Tonkin, and M. B. Eisen, "Noncanonical compensation of zygotic X transcription in early Drosophila melanogaster development revealed through single-embryo RNA-seq," PLoS Biology, vol. 9, Article ID e1000590, 2011.

[105] L. Rastelli and M. I. Kuroda, "An analysis of maleless and histone $\mathrm{H} 4$ acetylation in Drosophila melanogaster spermatogenesis," Mechanisms of Development, vol. 71, no. 1-2, pp. 107-117, 1998.

[106] P. Stenberg and J. Larsson, "Buffering and the evolution of chromosome-wide gene regulation," Chromosoma, vol. 120, no. 3, pp. 213-225, 2011.

[107] F. N. Hamada, P. J. Park, P. R. Gordadze, and M. I. Kuroda, "Global regulation of X chromosomal genes by the MSL complex in Drosophila melanogaster," Genes and Development, vol. 19, no. 19, pp. 2289-2294, 2005.

[108] X. Deng and V. H. Meller, "roX RNAs are required for increased expression of X-linked genes in Drosophila melanogaster males," Genetics, vol. 174, no. 4, pp. 1859-1866, 2006.

[109] B. Vicoso and B. Charlesworth, "Evolution on the X chromosome: unusual patterns and processes," Nature Reviews Genetics, vol. 7, no. 8, pp. 645-653, 2006.

[110] T. A. Gurbich and D. Bachtrog, "Gene content evolution on the X chromosome," Current Opinion in Genetics and Development, vol. 18, no. 6, pp. 493-498, 2008.

[111] M. J. Lercher, A. O. Urrutia, and L. D. Hurst, "Evidence that the human $\mathrm{X}$ chromosome is enriched for male-specific but not female-specific genes," Molecular Biology and Evolution, vol. 20, no. 7, pp. 1113-1116, 2003.

[112] D. Sturgill, Y. Zhang, M. Parisi, and B. Oliver, "Demasculinization of X chromosomes in the Drosophila genus," Nature, vol. 450, no. 7167, pp. 238-241, 2007. 
[113] L. M. Mikhaylova and D. I. Nurminsky, "Lack of global meiotic sex chromosome inactivation, and paucity of tissuespecific gene expression on the Drosophila X chromosome," BMC Biology, vol. 9, article 29, 2011.

[114] P. Stenberg, L. E. Lundberg, A. M. Johansson, P. Rydén, M. J. Svensson, and J. Larsson, "Buffering of segmental and chromosomal aneuploidies in Drosophila melanogaster," PLoS Genetics, vol. 5, no. 5, Article ID e1000465, 2009. 

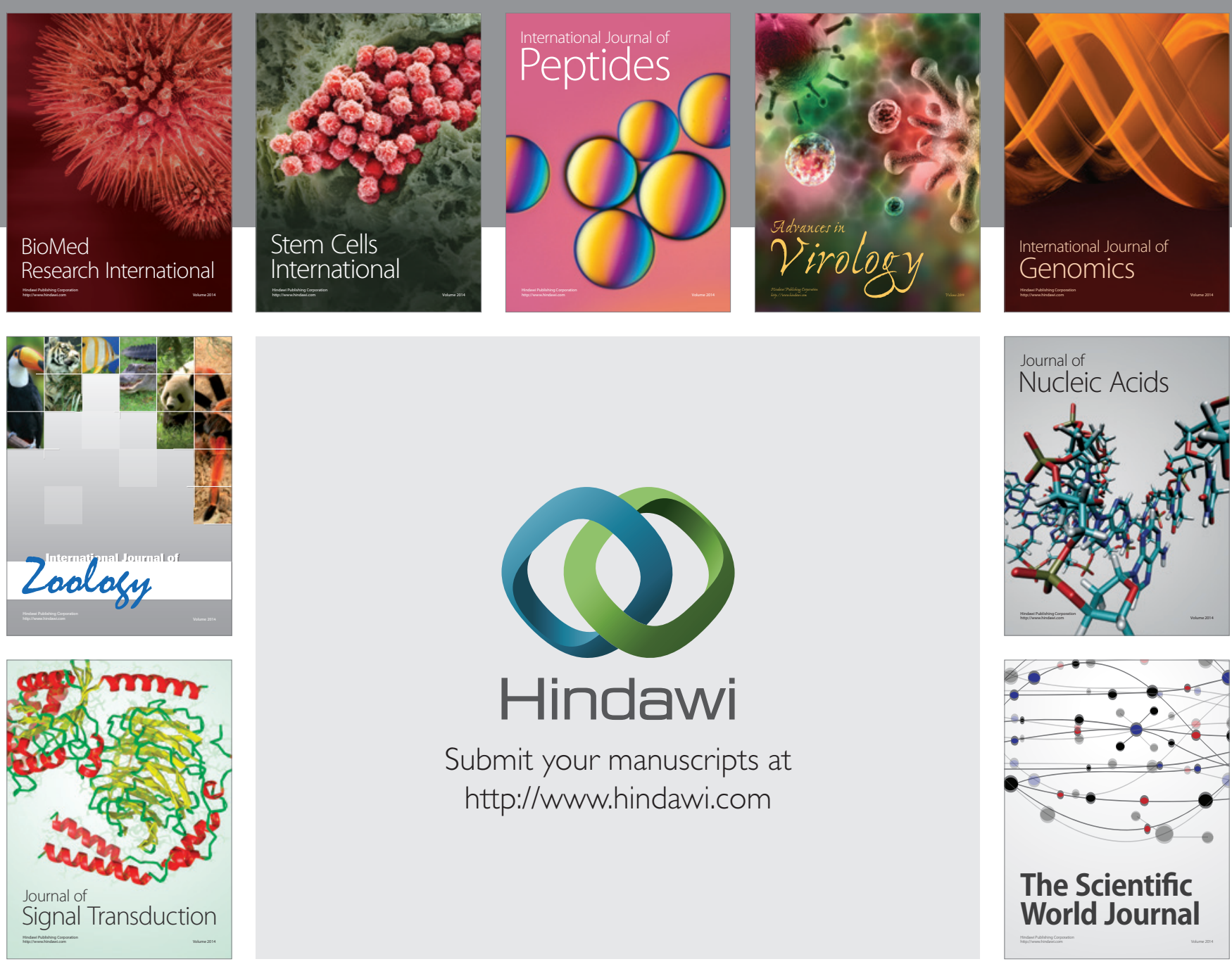

Submit your manuscripts at

http://www.hindawi.com
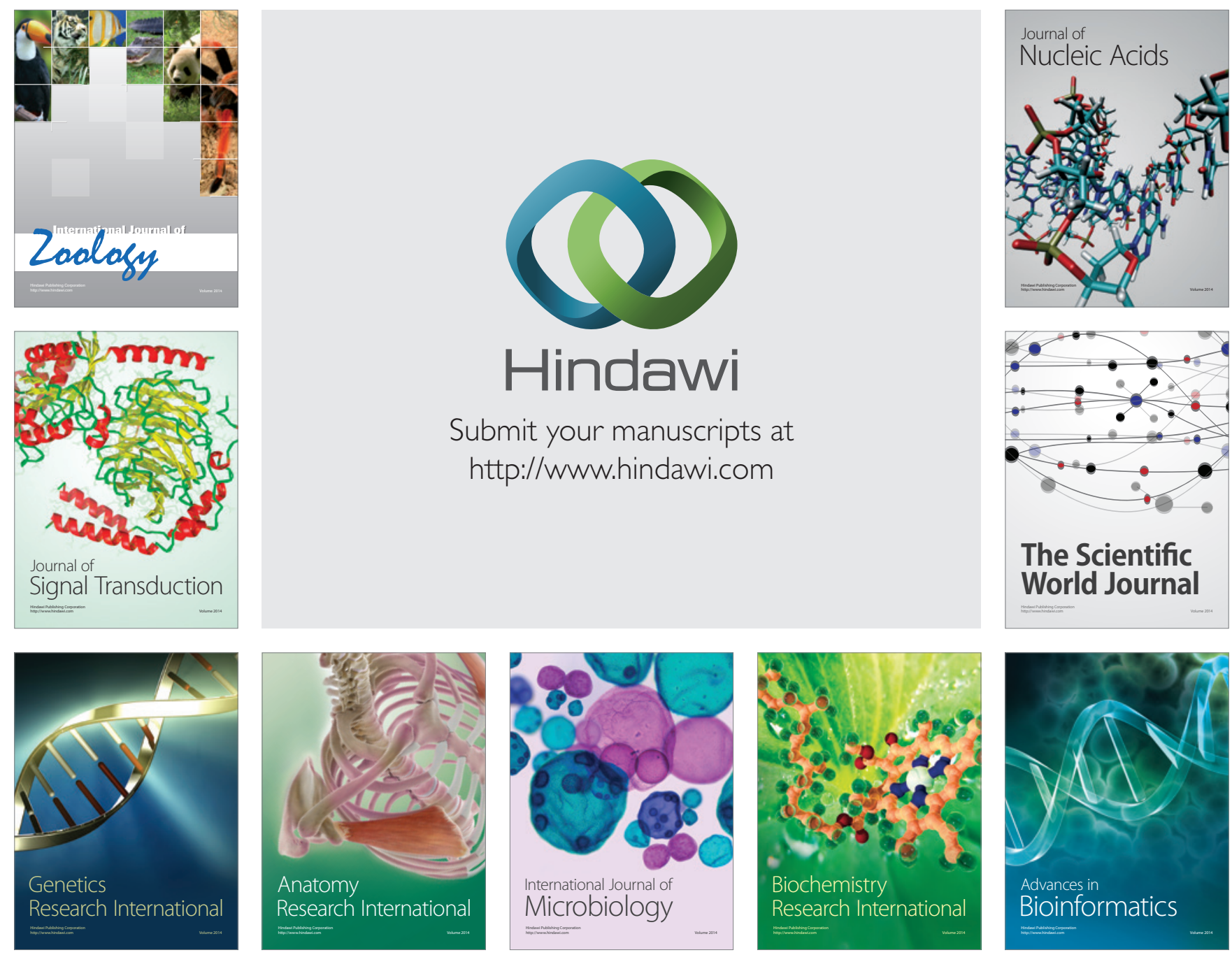

The Scientific World Journal
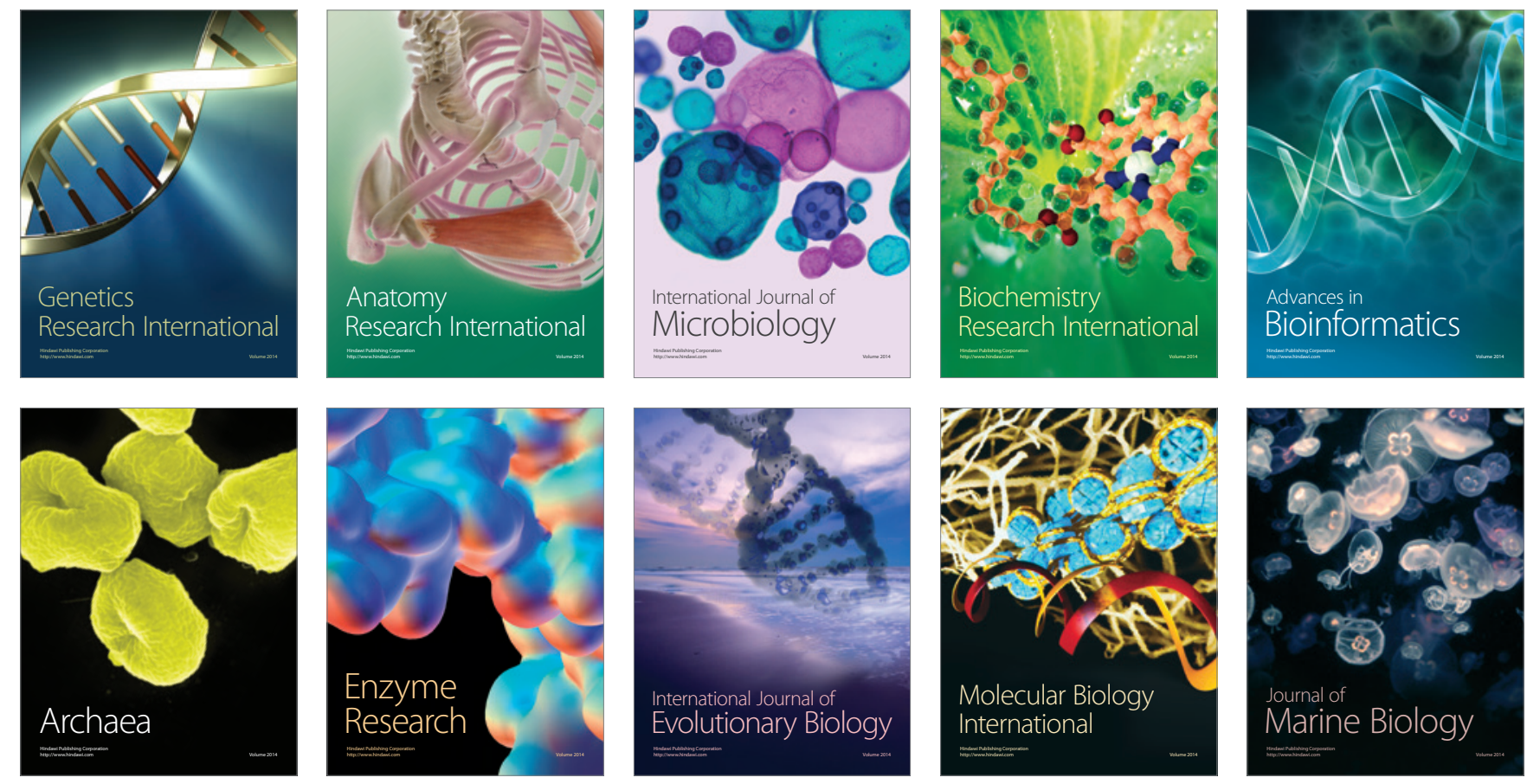